\title{
Surface-Water Monitoring in Watersheds of the Powder River Basin, 2005
}

\section{Powder River Basin Interagency Working Group}

The Powder River Basin (PRB) is a geologic structural basin that contains an extensive natural gas resource associated with regional coal deposits. This coalbed natural gas (CBNG) is located beneath millions of acres of private and public land in southeastern Montana and northeastern Wyoming (fig. 1). The PRB Interagency Working Group (IWG) was established in June 2003 as a forum to identify, discuss, and find solutions to issues of common concern to government agencies involved in permitting and monitoring CBNG development. The PRB IWG is led by the Bureau of Land Management (BLM) and is composed of managers and technical staff from local, State, tribal, and federal government agencies with land management, conservation, or regulatory responsibilities in the PRB, as well as agencies like the U.S. Geological Survey (USGS) that provide technical support.

The mission of the PRB IWG is to: (1) provide for environmentally sound energy development, (2) develop coordinated and complementary best management practices, guidelines, and programs related to CBNG activities to conserve and protect resources, (3) monitor the impact of CBNG activities and assess the effectiveness of mitigating measures, (4) develop and integrate the databases and scientific studies needed for effective resource management and planning, and to make that information readily available, and (5) promote compatibility in the application of each agency's mission.

In order to more effectively address the technical issues presented by CBNG development, Task Groups that are staffed by technical specialists from the member agencies of the PRB

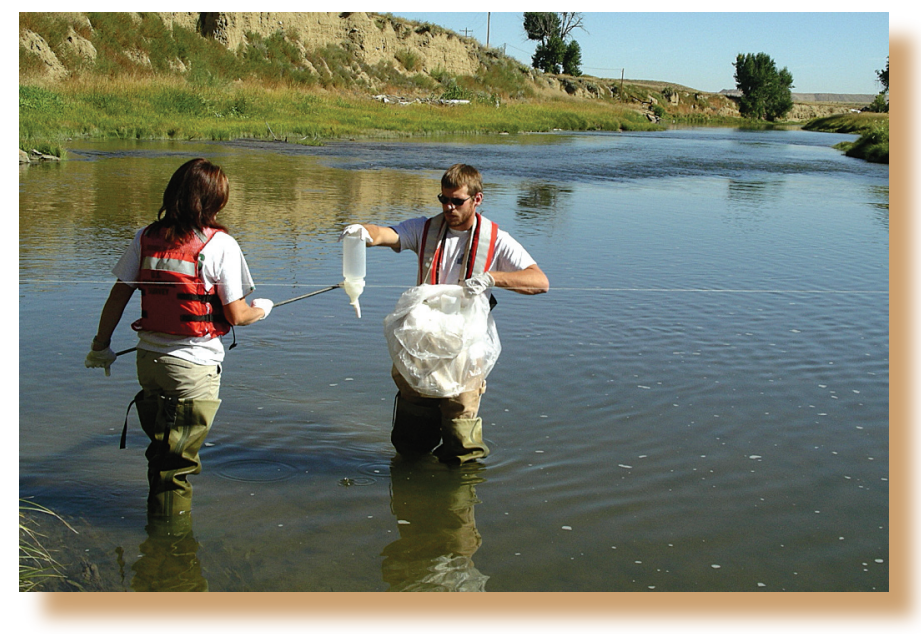

IWG were formed to address specific resource issues. The Task Groups include Air, Aquatics, Water, and Wildlife. More information about the PRB IWG and Task Group activities is available at URL http://www.wy.blm.gov/bfo/prbgroup/index.htm.

\section{Water Task Group}

Substantial volumes of ground water are extracted from coalbeds in order to produce CBNG. The removal of ground water from aquifers and use or disposal of produced water on the surface have the potential to cause environmental impacts. One objective of the Water Task Group is to develop and implement monitoring plans for surface water and ground water at local and regional scales. This monitoring will help agencies make more informed decisions regarding CBNG permitting, and allow for dissemination of information to the public. This factsheet summarizes the surface-water-monitoring plan developed by the Water Task Group and describes the surface-water monitoring accomplished during 2005.

\section{Surface-Water-Monitoring Plan}

The surface-water-monitoring plan is a proposed sampling network that is generally composed of sites where PRB IWG member agencies have been conducting surface-water monitoring. Sampling sites may be located on mainstems or selected tributaries in each watershed (fig. 1, table 1). Proposed sampling frequencies vary with stream type and constituent class (table 2). The constituent classes recommended for monitoring include:

- Streamflow

- Field measurements- $\mathrm{pH}$, dissolved oxygen, specific conductance, and temperature

- Major ions-dissolved calcium, magnesium, potassium, sodium, alkalinity, chloride, fluoride, sulfate, and silica; dissolved solids; and sodium-adsorption ratio

- Nutrients - total and dissolved nitrogen and phosphorus species

- Trace elements (primary) — total and dissolved aluminum, arsenic, barium, beryllium, iron, manganese, and selenium

- Trace elements (secondary) — total and dissolved cadmium, copper, chromium, lead, nickel, and zinc.

- Suspended sediment 


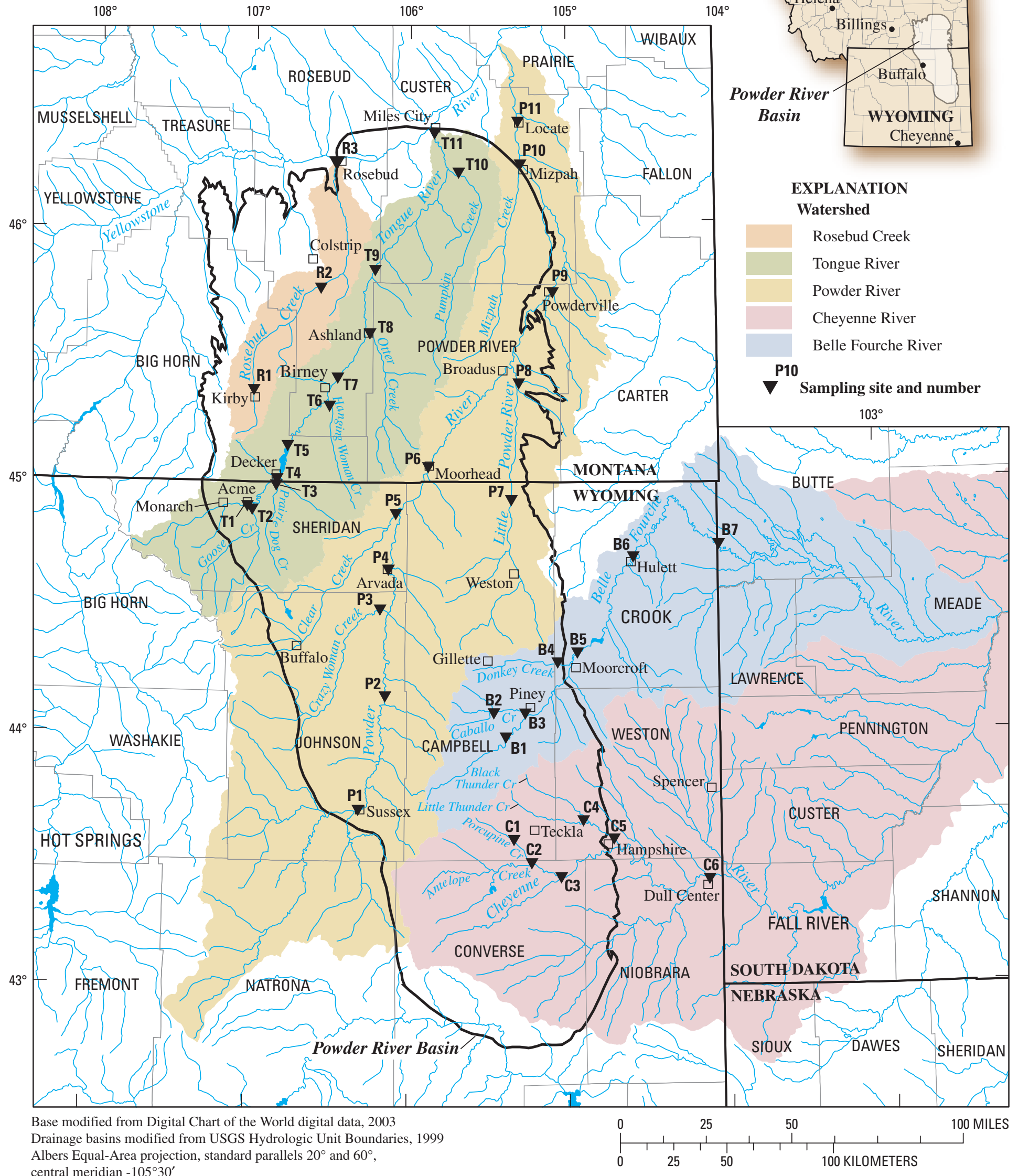

Albers Equal-Area projection, standard parallels $20^{\circ}$ and $60^{\circ}$,

Figure 1. Location of sampling sites proposed in the Water Task Group's surface-water-monitoring plan for the Powder River Basin. 
Table 1. Sampling sites proposed in the Water Task Group's surface-watermonitoring plan for the Powder River Basin.

\begin{tabular}{|c|c|c|c|}
\hline $\begin{array}{c}\text { Map } \\
\text { number }\end{array}$ & $\begin{array}{c}\text { U.S. } \\
\text { Geological } \\
\text { Survey } \\
\text { site number }\end{array}$ & Site name & $\begin{array}{l}\text { Stream } \\
\text { type }\end{array}$ \\
\hline R1 & 06295113 & $\begin{array}{l}\text { Rosebud Creek at reservation boundary near Kirby, } \\
\text { Mont. }\end{array}$ & Mainstem \\
\hline R2 & 06295250 & Rosebud Creek near Colstrip, Mont. & Mainstem \\
\hline R3 & 06296003 & Rosebud Creek at mouth, near Rosebud, Mont. & Mainstem \\
\hline $\mathrm{T} 1$ & 06299980 & Tongue River at Monarch, Wyo. & Mainstem \\
\hline $\mathrm{T} 2$ & 06305700 & Goose Creek near Acme, Wyo. & Tributary \\
\hline $\mathrm{T} 3$ & 06306250 & Prairie Dog Creek near Acme, Wyo. & Tributary \\
\hline $\mathrm{T} 4$ & 06306300 & Tongue River at State line near Decker, Mont. & Mainstem \\
\hline T5 & 06307500 & $\begin{array}{l}\text { Tongue River at Tongue River Dam, near Decker, } \\
\text { Mont. }\end{array}$ & Mainstem \\
\hline T6 & 06307600 & Hanging Woman Creek near Birney, Mont. & Tributary \\
\hline $\mathrm{T} 7$ & 06307616 & $\begin{array}{l}\text { Tongue River at Birney Day School Bridge, near } \\
\text { Birney, Mont. }\end{array}$ & Mainstem \\
\hline $\mathrm{T} 8$ & 06307740 & Otter Creek at Ashland, Mont. & Tributary \\
\hline T9 & 06307830 & $\begin{array}{l}\text { Tongue River below Brandenberg Bridge, near } \\
\text { Ashland, Mont. }\end{array}$ & Mainstem \\
\hline $\mathrm{T} 10$ & 06308400 & Pumpkin Creek near Miles City, Mont. & Tributary \\
\hline T11 & 06308500 & Tongue River at Miles City, Mont. & Mainstem \\
\hline $\mathrm{P} 1$ & 06313500 & Powder River at Sussex, Wyo. & Mainstem \\
\hline $\mathrm{P} 2$ & 06313605 & $\begin{array}{l}\text { Powder River below Burger Draw, near Buffalo, } \\
\text { Wyo. }\end{array}$ & Mainstem \\
\hline P3 & 06316400 & Crazy Woman at Upper Station, near Arvada, Wyo. & Tributary \\
\hline P4 & 06317000 & Powder River at Arvada, Wyo. & Mainstem \\
\hline P5 & 06324000 & Clear Creek near Arvada, Wyo. & Tributary \\
\hline P6 & 06324500 & Powder River at Moorhead, Mont. & Mainstem \\
\hline $\mathrm{P} 7$ & 06324970 & $\begin{array}{l}\text { Little Powder River above Dry Creek, near Weston, } \\
\text { Wyo. }\end{array}$ & Tributary \\
\hline P8 & 06325500 & Little Powder River near Broadus, Mont. & Tributary \\
\hline P9 & 06325650 & Powder River near Powderville, Mont. & Mainstem \\
\hline P10 & 06326300 & Mizpah Creek near Mizpah, Mont. & Tributary \\
\hline P11 & 06326500 & Powder River near Locate, Mont. & Mainstem \\
\hline $\mathrm{C} 1$ & 06364300 & Porcupine Creek near Teckla, Wyo. & Tributary \\
\hline $\mathrm{C} 2$ & 06364700 & Antelope Creek near Teckla, Wyo. & Tributary \\
\hline $\mathrm{C} 3$ & 06365900 & Cheyenne River near Dull Center, Wyo. & Mainstem \\
\hline $\mathrm{C} 4$ & 06375600 & Little Thunder Creek near Hampshire, Wyo. & Tributary \\
\hline $\mathrm{C} 5$ & 06376300 & Black Thunder Creek near Hampshire, Wyo. & Tributary \\
\hline $\mathrm{C} 6$ & 06386500 & Cheyenne River near Spencer, Wyo. & Mainstem \\
\hline B1 & 06425720 & $\begin{array}{l}\text { Belle Fourche River below Rattlesnake Creek near } \\
\text { Piney, Wyo. }\end{array}$ & Mainstem \\
\hline B2 & 06425800 & Caballo Creek near Gillette, Wyo. & Tributary \\
\hline B3 & 06425900 & Caballo Creek at mouth, near Piney, Wyo. & Tributary \\
\hline B4 & 06426400 & Donkey Creek near Moorcroft, Wyo. & Tributary \\
\hline B5 & 06426500 & Belle Fourche River below Moorcroft, Wyo. & Mainstem \\
\hline B6 & 06428050 & Belle Fourche River below Hulett, Wyo. & Mainstem \\
\hline B7 & 06428500 & $\begin{array}{l}\text { Belle Fourche River at Wyoming-South Dakota } \\
\text { State line }\end{array}$ & Mainstem \\
\hline
\end{tabular}

Table 2. General sampling strategy proposed in the Water Task Group's surface-water-monitoring plan for the Powder River Basin.

\begin{tabular}{cll}
$\begin{array}{c}\text { Stream } \\
\text { type }\end{array}$ & Sampling frequency & \multicolumn{1}{c}{ Constituent class } \\
\hline Mainstem & Continuous & Streamflow \\
& 12 times per year & Field measurements \\
& 12 times per year & Major ions \\
& 2 times per year & Nutrients \\
& 12 times per year & Trace elements, primary \\
& 2 times per year & Trace elements, secondary \\
& 12 times per year & Suspended sediment \\
& & \\
Tributary & Continuous & Streamflow \\
& 6 times per year & Field measurements \\
6 times per year & Major ions \\
2 times per year & Nutrients \\
6 times per year & Trace elements, primary \\
2 times per year & Trace elements, secondary \\
6 times per year & Suspended sediment \\
\hline
\end{tabular}

\section{Monitoring Summary, 2005}

Because of funding shortfalls for surface-water monitoring, only part of the proposed sampling in the surface-water-monitoring plan was accomplished during 2005 (table 3). For the sites where the sampling was partially completed, either the sampling frequency was less than the proposed sampling frequency or not all of the constituent classes were analyzed. The Tongue River watershed was the only watershed where the sampling proposed in the surface-water-monitoring plan was fully completed.

Several of the agencies that participate on the PRB IWG contributed funding for monitoring and reporting, including:

- BLM,

- Montana Department of Environmental Quality,

- Montana Department of Natural Resources and Conservation,

- Northern Cheyenne Tribe,

- U.S. Environmental Protection Agency,

- USGS,

- Wyoming Department of Environmental Quality, and the

- Wyoming State Engineer's Office.

Streamflow data and water-quality samples were collected by USGS personnel using standard USGS field methods (http://water.usgs.gov/owq/FieldManual/). Samples were analyzed at the USGS National Water Quality Laboratory in Lakewood, Colorado. 
Table 3. Monitoring accomplished for surface-water-monitoring plan during 2005.

, completed; $\bigcirc$, partially completed; and $\bigcirc$, not completed.]

\begin{tabular}{|c|c|c|c|c|c|c|c|}
\hline $\begin{array}{c}\text { Map } \\
\text { number }\end{array}$ & $\begin{array}{c}\text { Stream- } \\
\text { flow }\end{array}$ & $\begin{array}{c}\text { Field } \\
\text { measure- } \\
\text { ments }\end{array}$ & $\begin{array}{c}\text { Major } \\
\text { ions }\end{array}$ & Nutrients & $\begin{array}{c}\text { Trace } \\
\text { elements, } \\
\text { primary }\end{array}$ & $\begin{array}{c}\text { Trace } \\
\text { elements, } \\
\text { second- } \\
\text { ary }\end{array}$ & $\begin{array}{c}\text { Sus- } \\
\text { pended } \\
\text { sediment }\end{array}$ \\
\hline $\mathrm{R} 1$ & & 0 & 0 & 0 & 0 & 0 & 0 \\
\hline $\mathrm{R} 2$ & ? & 0 & $\bigcirc$ & 0 & $\bigcirc$ & 0 & $\bigcirc$ \\
\hline $\mathrm{R} 3$ & & 0 & 0 & 0 & 0 & 0 & $\bigcirc$ \\
\hline $\mathrm{T} 1$ & & & & 0 & 0 & O & 0 \\
\hline $\mathrm{T} 2$ & & & & $\mathrm{C}$ & 0 & & 0 \\
\hline $\mathrm{T} 3$ & & & & 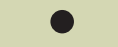 & $\mathrm{C}$ & & 0 \\
\hline $\mathrm{T} 4$ & & & & 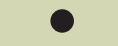 & C & 0 & 0 \\
\hline T5 & & & & 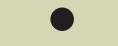 & 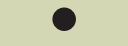 & 0 & 0 \\
\hline T6 & & & & 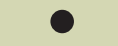 & 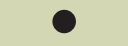 & 0 & 0 \\
\hline $\mathrm{T} 7$ & & & & 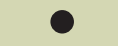 & 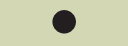 & 0 & 0 \\
\hline $\mathrm{T} 8$ & & & & 0 & 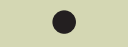 & C & 0 \\
\hline T9 & & & & 0 & $\mathrm{C}$ & $\mathrm{C}$ & 0 \\
\hline $\mathrm{T} 10$ & & & & $\mathrm{C}$ & 0 & 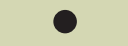 & 0 \\
\hline $\mathrm{T} 11$ & & 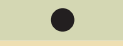 & 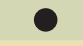 & 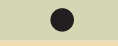 & O & 0 & 0 \\
\hline $\mathrm{P} 1$ & 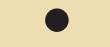 & 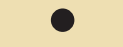 & 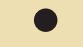 & $\bigcirc$ & $\bigcirc$ & $\bigcirc$ & $\bigcirc$ \\
\hline $\mathrm{P} 2$ & $\bigcirc$ & C & 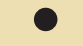 & $\bigcirc$ & $\bigcirc$ & $\bigcirc$ & $\bigcirc$ \\
\hline P3 & & 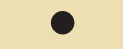 & $C$ & 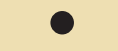 & $\bigcirc$ & $\bigcirc$ & O \\
\hline $\mathrm{P} 4$ & & 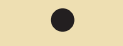 & $C$ & $\bigcirc$ & $\bigcirc$ & $\bigcirc$ & $\bigcirc$ \\
\hline P5 & & 8 & 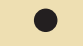 & $\bigcirc$ & $\bigcirc$ & 0 & $\bigcirc$ \\
\hline P6 & & & & & 0 & 0 & 0 \\
\hline $\mathrm{P} 7$ & & & & & $\bigcirc$ & $\bigcirc$ & 0 \\
\hline P8 & $\bigcirc$ & & & & 0 & 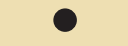 & 0 \\
\hline P9 & 0 & 0 & 0 & $\bigcirc$ & $\bigcirc$ & $\bigcirc$ & $\bigcirc$ \\
\hline $\mathrm{P} 10$ & $\bigcirc$ & 0 & $\bigcirc$ & $\bigcirc$ & $\bigcirc$ & 0 & $\bigcirc$ \\
\hline P11 & & & & 0 & 0 & & 0 \\
\hline $\mathrm{C} 1$ & & & & $\bigcirc$ & $\bigcirc$ & $\bigcirc$ & $\bigcirc$ \\
\hline $\mathrm{C} 2$ & $\bigcirc$ & 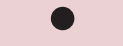 & & $\bigcirc$ & $\bigcirc$ & $\bigcirc$ & $\bigcirc$ \\
\hline C3 & & & & $\bigcirc$ & $\bigcirc$ & $\bigcirc$ & $\bigcirc$ \\
\hline $\mathrm{C} 4$ & $\bigcirc$ & & & $\bigcirc$ & $\bigcirc$ & $\bigcirc$ & $\bigcirc$ \\
\hline $\mathrm{C} 5$ & 0 & & & $\bigcirc$ & $\bigcirc$ & 0 & 0 \\
\hline C6 & & & 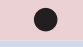 & 0 & $\bigcirc$ & $\bigcirc$ & $\bigcirc$ \\
\hline B1 & $\bigcirc$ & 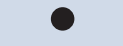 & 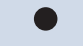 & $\bigcirc$ & $\bigcirc$ & $\bigcirc$ & 0 \\
\hline B2 & $\bigcirc$ & & $\Omega$ & 0 & $\bigcirc$ & $\bigcirc$ & 0 \\
\hline B3 & $\bigcirc$ & & & $\bigcirc$ & $\bigcirc$ & $\bigcirc$ & 0 \\
\hline B4 & $\bigcirc$ & 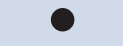 & & $\bigcirc$ & $\bigcirc$ & 0 & 0 \\
\hline B5 & & & & 0 & $\bigcirc$ & 0 & 0 \\
\hline B6 & $\bigcirc$ & & & 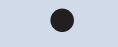 & $\bigcirc$ & $\bigcirc$ & 0 \\
\hline B7 & 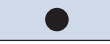 & 0 & 0 & 0 & 0 & 0 & 0 \\
\hline
\end{tabular}

\section{Data Availability}

Data collected as part of Water Task Group surface-water-monitoring plan are stored electronically in the USGS National Water Information System. Continuous streamflow and water-quality data are available to the public at URL: http://waterdata.usgs.gov/nwis/. Other USGS data for Montana and Wyoming can be accessed at http://mt.water.usgs.gov/, http://tonguerivermonitoring.cr.usgs.gov/, and http://wy.water.usgs.gov/.

\section{Future Work}

Another objective of the Water Task Group is to interpret the surface-water-monitoring data that are collected. Until more data are collected, much of the initial interpretive analysis may focus on sites with historical data that were collected for previous monitoring programs. For example, the Powder River at Arvada, Wyoming has been sampled for many years, and relations between constituents, such as specific conductance and the sodium-adsorption ratio, have been established (fig. 2). If the monitoring data indicate that water quality is changing, managers can use adaptive management and appropriate mitigation measures to address environmental concerns.

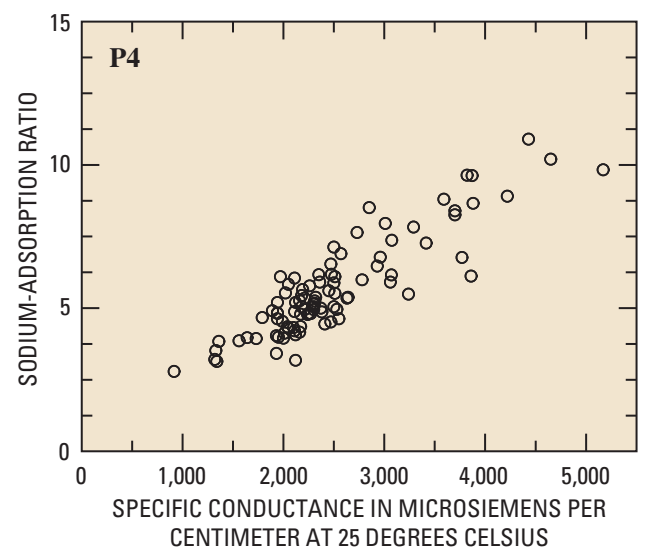

Figure 2. Specific conductance and sodium-adsorption ratio relation for the Powder River at Arvada, Wyo.

\section{For more information, contact:}

Water Science Center Director,

U.S. Geological Survey

Montana Water Science Center

3162 Bozeman Avenue

Helena, Montana 59601

Water Science Center Director,

U.S. Geological Survey

Wyoming Water Science Center

2617 E. Lincolnway, Suite B

Cheyenne, Wyoming 82001

Field Office Manager,

Bureau of Land Management

Buffalo Field Office

1425 Fort Street

Buffalo, Wyoming 82834-2436

Field Office Manager,

Bureau of Land Management

Miles City Field Office

111 Garryowen Road

Miles City, Montana 59301

By Melanie L. Clark ${ }^{\text {, }}$

John H. Lambing ${ }^{1}$,

and Andrew L. Bobst ${ }^{2}$

${ }^{1}$ U.S. Geological Survey

${ }^{2}$ Bureau of Land Management

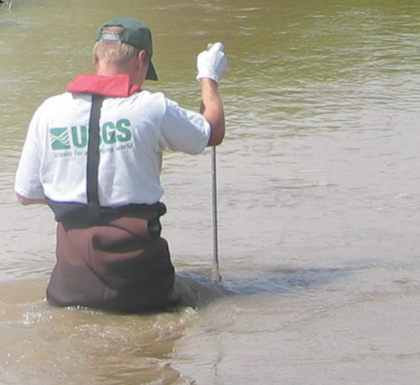

